\title{
2017 Thank you to our reviewers
}

The Editor would like to publicly acknowledge the people listed below who served as reviewers on the journal during 2017. Without their efforts, the quality of the journal could not be sustained.

Ajmonemarsan, Nina

Alfonso, Fernando

Al-Janabi, Ali Abdul

Hussein S.

Ashraf, Rizwan

Atar, Dan

Avolio, Alberto

Bailly, Sebastien

Baron, Suzanne

Barros De Oliveira Sa,

Michel Pompeu

Bedossa, Marc

Bellows, Brandon

Beltowski, Jerzy

Benfari, Giovanni

Biondizoccai, Giuseppe

Bonhomme, Fanny

Borghi, Claudio

Brown, Adam

Buckley, John

Bull, Russel

Bulluck, Heerajnarain

Bunch, Thomas

Cademartiri, F.

Canpolat, Ugar

Carrié, Didier

Chin, Paul

Chirillo, Fabio

Christian, Susan

Cupples, Margaret

Currie, C. J.

Dall, Christian H.

Darbar, Dawood

Dascenzo, Fabrizio

De Backer, Ole

De Boer, Rudolf

Delewi, Ronak

Donal, Erwan

Dong, Zhao

Dos Santos, Catarina

Rodrigues

Durante, Alessandro

Eccleshall, Simon

Efthimiadis, Georgios K.

Frits, Rosendaal
Frohlich, Georg

Gajewski, Byron J.

Gall, Nicholas

Girardin, Francois

Gotberg, Matthias

Greve, Anders M.

Hamilos, Michalis

Hamilton-Craig,

Christian R.

Huntley, Alyson L.

Indermuehle, Andreas

Ishii, Hideki

Isshiki, Takaaki

Iung, Bernard

Izawa, Atsushi

Jeong, Young-Hoon

Jia, Youhong

Jin, Hongfang

Kang, Duk Hyun

Karimi, Ashkan

Kato, Johji

Kato, Masahiko

Kaya, Hasan

Keller, Frieder

Kharbanda, Rajesh

Khoja, Adeel

Knapp, Guido

Kojonazarov, Baktybek

Kones, Richard

Konstantinides, Stavros

Korosoglou, Grigorios

Krahn, Andrew D.

Kramer, Robert

Kreutz, Rolf

Kreutz, Rolf P.

Kurisu, Satoshi

Kuusisto, Johanna

Ladwig, Karl-Heinz

Lavie, Carl

Lawler, P

Lee, Kwan

Lenzen, Mattie

Leslie, Stephen

Lester, Will

Lim, Sern
Liu, Songtao

Loffredo, Lorenzo

Ludman, Peter

Lyons, Kristopher S.

Macnab, Anita

Mager, Diana R.

Mewton, Nathan

Min, Pil-Ki

Mizariene, Vaida

Moore, John T.

Morgan, Drake

Morishita, Ryuichi

Muthu, Sivakumar

Nalini, Mahdi

Newby, David

Nielsen, Forrest H.

Nielsen, Peter Bronnum

Nijjer, Sukhjinder S.

Niswender, K.

Okoshi, Marina P.

Orban, Martin

Packard, René

Panchal, Hemang B.

Park, Chi Hye

Park, Seong Wook

Peix, Amalia

Piek, Jan

Plank, Fabian

Pourafkari, Leili

Puddu, Paolo Emilio

Räber, Lorenz

Raftos, Julia E.

Raj, Satish

Raja, Shahzad

Ramadi, Ailar

Reid, Ian

Reynolds, T.

Ribera, Aida

Rigamonti, Fabio

Roberts, Lara N.

Roche, Frederic

Roditi, Giles

Roh, Young Sook

Romero-Farina, G.

Rosanoff, Andrea
Rossano, Joseph

Sabaté, Manel

Sandfort, Veit

Sasson, Comilla

Savion, Naphtali

Schoenenberger, Andreas

Seiffert, Moritz

Serebruany, Victor

Shambrook, James

Silva, Matthew

Simopoulos, Artemis P.

Skinner, Jonathan

Sohal, Manav

Steeds, Richard

Stoner, Lee

Sutanto, Hendri

Suzuki, Yoriyasu

Swaans, M. J.

Tada, Tomohisa

Tanaka, Hidekazu

Teiger, Emmanuel

Teres, Cheryl

Thota, Anil

Tomizawa, Nobuo

Trifunovic, Zoran

Turner, David

Unger, Jeff

Vachiery, Jean-Luc

Van Smeden, Maarten

Vishram, Julie K. K.

Vitarelli, Antonio

Von Bonsdorff,

Mikaela B.

Wang, Tzung-Dau

Watson, Timothy

West, Robert

Wilson, Lance D.

Winchester, David E.

$\mathrm{Wu}$, Alan

$\mathrm{Xu}, \mathrm{W}$.

Yan, Xiaoxiang

Yang, Eric

Yoshida, Kentaro

Zaremba, Tomas

Zhou, Zi-Hua 